\title{
The Genomic and Structural Organization of SARS-CoV-2: A Mutational Perspective
}

\author{
Mohammad K. Parvez ${ }^{1 *}$, Sakina Niyazi ${ }^{2}$ \\ ${ }^{1}$ Department of Pharmacognosy, King Saud University College of Pharmacy, Riyadh 11451, Saudi Arabia. \\ ${ }^{2}$ Jaypee Wishtown, Sector 128, Noida 201304, Uttar Pradesh, India.
}

\begin{abstract}
The ongoing pandemic due to the novel SARS-CoV-2 disease (COVID-19) has exerted a great toll on human health. The SARS-CoV-2 is the third most pathogenic human CoV after SARS-CoV-1 and MERS-CoV, which is classified within the genus Betacoronavirus. Though the actual source of its origin and transmission is still unclear, genetic analysis has shown its very close similarity $(\sim 96 \%)$ with bat SARS-like CoV. SARS-CoV-2 is a spherically-icosahedral virus with a plus-sense single-strand RNA ( $\sim 30 \mathrm{~kb})$ genome defined into thirteen open reading frames, which encode 2 non-structural polyproteins, 4 structural proteins and 6 accessory proteins. Of its structural proteins the ' $\mathrm{S} 1$ ' subunit of spike (S) contains the cellular ACE-2 receptor binding domain (RBD) whereas the 'S2' subunit is required for cell membrane fusion. The membrane (M) protein participates in cell-fusion whereas envelope (E) is necessary for virion assembly and morphogenesis. The non-structural polyproteins (ppla and $\mathrm{pp} 1 \mathrm{~b}$ ) undergo proteolytic processing to produce a total of 16 small proteins, which are involved in mRNA synthesis and replication. Of the accessory proteins ( $3 \mathrm{a}, 6,7 \mathrm{a}, 7 \mathrm{~b}, 8$ and $9 \mathrm{~b})$, few are known to modulate host-innate immunity. Interestingly, ' $3 b$ ' is absent in SARS-CoV-2 that significantly differentiates it from other human CoV. Detection of several novel mutations in ' $3 a$ ', ' $3 b$ ' and 'ORF8' proteins, notably in the ' $\mathrm{S}$ ' RBD strongly suggest SARS-CoV-2 enhanced cell attachment and facilitated entry, its high infectivity and disease severity in humans. The recent emergence of highly contagious SARS-CoV-2 RBD variants in the United Kingdom (B.1.1.7 strain), South Africa (B.1.351 strain) and Brazil (P.1 strain), and their subsequent spread to other counties have raised serious concerns.
\end{abstract}

Keywords: Coronavirus; Severe Acute Respiratory Syndrome; SARS-CoV-2; COVID-19; ACE-2; Mutations.

\section{Introduction}

In the recent decades, incidences of devastating viral epidemics have marked the Asia-Pacific region as the global hot-spot for emerging new pathogenic viruses [1]. The novel severe acute respiratory syndrome coronavirus-2 (SARSCoV-2) disease or COVID-19 pandemic first reported in China in Dec. 2019, has affected nearly 114 million people, with over 2.5 million deaths worldwide [2-4]. SARS-CoV-2 is the seventh human infecting and third highly pathogenic human CoV after the SARS-CoV-1 and Middle-East respiratory syndrome CoV (MERS-CoV) [4, 5]. The human-to-human direct transmission of SARS-CoV-2 through multiple modes, such as nasal droplets, oral mucus and aerosols, including fomites has been confirmed [6,7]. SARS-CoV-2 has an incubation period of 2-14 days with symptoms of cough, fever, headache and shortness of breath, which may progress to mild-to-severe pneumonia and death $[4,8]$. COVID-19 patients, mostly of old age group or with comorbidity like pulmonary, cardiac, renal or

\footnotetext{
* Corresponding author: khalid_parvez@yahoo.com
}

\section{doi) http://dx.doi.org/10.28991/SciMedJ-2021-0301-8}

$>$ This is an open access article under the CC-BY license (https://creativecommons.org/licenses/by/4.0/).

(C) Authors retain all copyrights. 
hepatic disorders have shown higher mortality rate than those with pneumonia only. Among COVID-19 positive cases, about $80 \%$ individuals remain asymptomatic, $15 \%$ with hospitalization and $5 \%$ develop acute respiratory distress syndrome (ARDS) or multi-organ failure [9]. In addition, a good proportion of COVID-19 patients have also shown evidences of digestive and hepato-biliary symptoms, where high amounts of SARS-CoV-2 genomic RNA has been detected in gastrointestinal, rectal and stool specimen [10-12]. Further detection of SARS-CoV-2 RNA in municipal or waste water samples has suggested its plausible fecal-oral transmission through contaminated water [13].

In general, human CoV do not cause life-threatening disease. However, because of the zoonotic origin of SARSCoV-2 similar to SARS-CoV-1, humans lack pre-existing natural immunity i.e. 'herd-immunity' against it [14]. In naïve population therefore, exposure to SARS-CoV-2 leads to a much delayed time to develop adaptive-immune responses. Also, unlike SARS-CoV-1, most of the spread of SARS-CoV-2 occurs through asymptomatic infection [15-17], which is a bottleneck for its quick containment. Moreover, unlike SARS-CoV-1 and MERS-CoV, the precise mechanism of modulation of host-innate immune responses and severe pathogenesis by SARS-CoV still remains elusive [18]. Nonetheless, within a year of COVID-19 health crisis, we have a remarkable understanding of its epidemiology, clinical presentations, immune-pathobiology, treatments and preventive strategies. Currently plasma or antibody based rapid-test kits and reverse-transcription polymerase chain reaction (RT-PCR) are the routine diagnostic tools to identify COVID-19 positive cases. In the absence of specific therapeutics, several repurposed drugs are currently under clinical trials or approved for emergency use [19, 20]. Fortunately, of the leading vaccine candidates under final stages of trials, at least seven are now granted approval in some countries.

\section{SARS-CoV-2 genomic organization}

The novel SARS-CoV-2 together with SARS-CoV-1, MERS-CoV, HCoV-OC43 and HCoV-HKU1, have been classified within the genus Betacoronavirus. SARS-CoV-2 is a spherically-icosahedral and enveloped virus ranging from 80 to $120 \mathrm{~nm}$ [21]. It has a linear positive-sense, single-strand RNA ( 29.9 kb) genome that is 5'-capped and 3'polyadenylated, and transcribes into a set of several sub-genomic mRNAs with a common 5'-leader sequence [21]. SARS-CoV-2 genome is defined into 12 active open reading frames (ORFs; 5'-3') comprising of two non-structural, four structural and six accessory protein coding sequences. Genomic sequences and phylogenetic analysis of the isolated SARS-CoV-2 have revealed its very close similarity $(\sim 96 \%)$ with bat SARS-like-CoV (bat-SL-CoVZC45, bat-SL-CoVZXC21 and SL-CoV-RaTG13), indicating its zoonosis associated with horseshoe bats [22, 23]. The viral RNA genome consists of 13 open reading frames (ORFs), which encode four structural, two non-structural polyproteins producing sixteen processed products, and seven accessory proteins (Figure 1A) [24].

A. Genome organization

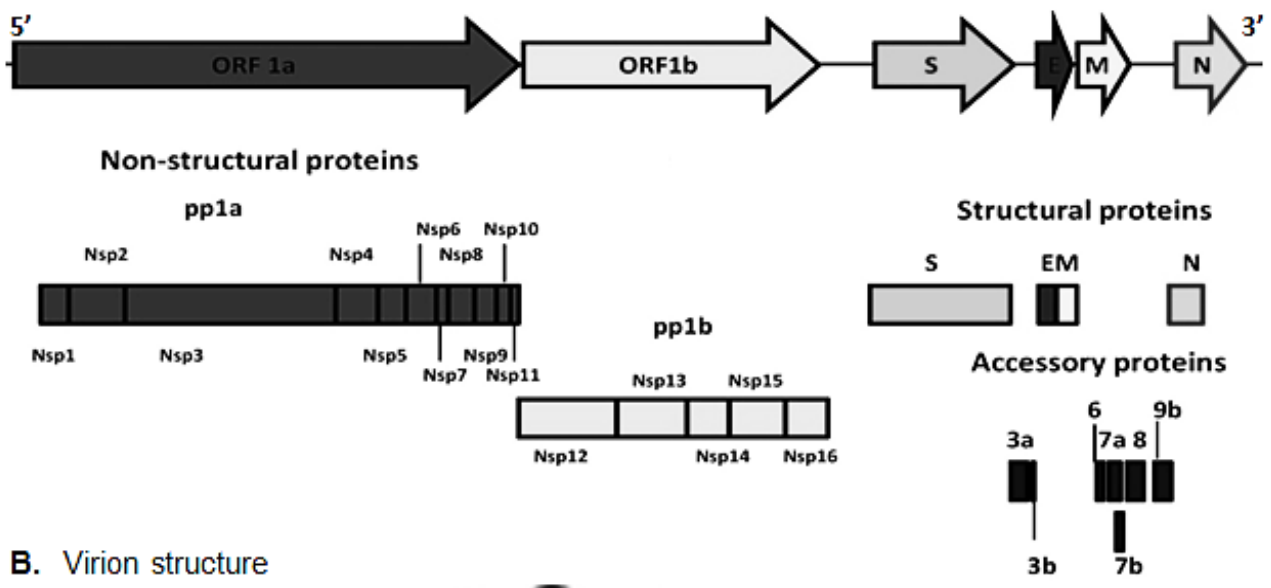

B. Virion structure

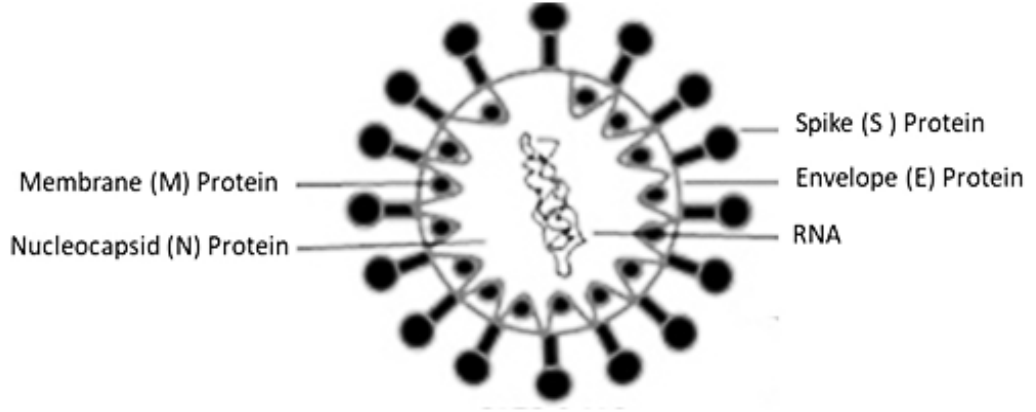

Figure 1. A graphic representation of the genomic and structural organization of SARS-CoV-2. (A) Genomic RNA showing the open reading frames $\left(5^{\prime}-3^{\prime}\right)$ encoding non-structural replicase polyproteins (pp1a and pp1b) and their processed products, structural proteins (spike, $S$; envelope, E; membrane, $M$; and nucleocapsid, $N$ ) and accessory proteins (3a, 3b, 6 , $7 a, 7 b, 8$ and 9b). (B) virion structure. Adapted and modified with permission [12, 20]. 


\section{Structural Proteins}

Of the four encoded structural proteins, the club or pear-shaped spike (S) proteins bind to cell receptor angiotensinconverting enzyme 2 (ACE2) whereas envelope (E) and membrane (M) proteins form the viral shell or envelope (Figure 1B). The nucleocapsid $(\mathrm{N})$ proteins form the viral capsid in which the genomic RNA is packaged. Notably, the characteristic structural projections known as hemagglutinin-esterase (HE) proteins located beneath the spikes in some betacoronaviruses are absent in SARS-CoV-2 [21]. The ' $\mathrm{S}$ ' protein is a large type I trans-membrane glycoprotein that is present on the outer surface of the virion, and gives a crown-like appearance that has earned it the name 'coronavirus' [23]. The SARS-CoV-2 'S' protein shares $\sim 76 \%$ sequence identity with that of SARS-CoV-1 and $\sim 80 \%$ identity with bat-SL-CoV [24, 25]. 'S' protein has two structural subunits (S1 and S2) where the 'S1' subunit contains the ACE2-binding domain (RBD) and the 'S2' subunit contains structural elements viz., a membrane fusion peptide (FP), an internal fusion peptide (IFP), two heptamer repeats (HR) and a trans-membrane domain (TM) required for cell membrane fusion [26]. Notably, the SARS-CoV-2 'S1'is highly variable with nearly $70 \%$ identity to those of batSL-CoV and SARS-CoV-1. In contrast, 'S2' is highly conserved and shares up to $99 \%$ identity with that of bat-SL$\mathrm{CoV}$ and SARS-CoV-1 [27]. Interestingly, the SARS-CoV-2 S1-S2 junction sequences contain a 'furin-like' cleavage site, as a result of 12-nucleotide insertion, not found in bat-SL-CoV and SARS-CoV-1 [27, 28]. In addition, of the six residues located in RBD, five differ between SARSCoV-2 and SARS-CoV-1, suggesting that the novel SARS-CoV-2 spike protein binds to ACE2 more strongly than that of SARS-CoV-1, hence making the virus more infectious to humans [27-30].

The ' $\mathrm{E}$ ' protein is a small glycoprotein that plays a critical role in the assembly and morphogenesis of virions within the infected cell [31]. The ' $\mathrm{M}$ ' protein is a trans-membrane glycoprotein and is involved in virion morphogenesis and maturation through interacting with other structural proteins [32]. The ' $\mathrm{N}$ ' is a phosphoprotein that is highly conserved among SARS-CoV-1 and bat-SL-CoV, and plays a crucial role in RNA encapsidation, capsid structure stabilization, replication and transcription. Recently, high-resolution crystal structure of SARS-CoV-2 nucleocapsid (N2b domain) has revealed its compact intertwined architecture and self-assembly properties very similar to that of SARSCoV-1 and MERS-CoV [33]. Though not established in COVID-19 patients, it has been reported to be highly antigenic in about $90 \%$ of SARS-CoV-1-infected individuals [34].

\section{Non-structural Proteins}

The non-structural replicase proteins are translated as two large polyproteins (pp1a and pp1b) that further undergo proteolytic cleavage to produce 16 individually active small proteins [35]. The 'pp1a' is processed to generate 11 proteins (nsp1-nsp11) whereas 'pp1b' produces 5 proteins (nsp12-16). Non-structural proteins of SARS-CoV-2 are involved in viral RNA transcription and replication, including modulation of host innate immunity [33]. However, the precise functions of nsp2 and nsp11 still remain elusive [36]. Due to insignificant differences between pp1a/b of SARS-CoV-2 and those of other betacoronaviruses, the pp1a/b of SARS-CoV-2 has not been thoroughly analyzed. Nonetheless, a 42 amino acid insertion in SARS-CoV-2 'ppla/b' papain-like protease region has been reported recently [37].

\section{Accessory Proteins}

In addition to the structural and non-structural proteins, seven accessory proteins $(3 \mathrm{a}, 3 \mathrm{~b}, 6,7 \mathrm{a}, 7 \mathrm{~b}, 8$ and $9 \mathrm{~b})$ are also synthesized by all betacoronaviruses, and they are mainly involved in countering host innate immune system. However, in a recent sequence analysis of different betacoronavirus genomic RNAs, SARS-CoV-2 has shown insertion of six stop codons leading to aborted translation of ' $3 b$ ' [28]. This observation has suggested the number of accessory proteins to be six instead of seven and therefore, consolidating the number of all synthesized proteins to twenty six. Also, SARS-CoV-2 significantly varies in the conserved aggregation motif (VLVVL), including deletion of the diacidic motif (XDE) within '3a' as compared to SARS-CoV-1, bat-SL-CoV and civet-SL-CoV [28, 37].

\section{Significant Mutations in SARS-CoV-2 Genes and Proteins}

Approximately $80 \%$ of human infecting viruses are zoonotic, which initially poorly adapt and replicates in a new host or cross species-barrier [1]. RNA viruses, due to the high replication-error rate ( $10-4$ error/site/cycle) of their RNA polymerase are more genetically diversified than DNA viruses [1]. Because SARS-CoV-2 is newly introduced to humans, we have limited knowledge on the mechanism(s) underlying its high infectivity and pathogenesis in humans. Nonetheless, ample of recent comparative RNA and protein sequence analyses has reported several mutations in different SARS-CoV-2 isolates, suggesting their crucial roles in high infection rates and disease severity [28, 39-44]. Most importantly, substitution mutations in SARS-CoV-2 ' $S$ ' RBD residues, including other unique mutations in $\mathrm{RBM}$ as well as introduction of furin-like sequences have been suggested for its strong binding with ACE-2 and high infectivity [27, 28, 44, 45]. Notably, proteolytic cleavage of ' $S$ ' protein at furin-site leads to its open conformation that gives advantage of enhanced binding to host ACE-2 [27]. This has been supported by neuropilin-1 mediated cleavage of SARS-CoV-2 furin towards its significant improvement in ACE-2 binding [46]. 
Very recently, comparative sequence analysis of the four structural and 'ORF8' proteins of 100 SARS-CoV-2 isolates of different countries has revealed thirteen substitutions and/or deletion in ' $\mathrm{S}$ ', three substitutions in ' $\mathrm{N}$ ', and one substitution in ' $M$ ' protein [47]. Another such study has reported frequent mutations in ' $S$ ', 'N', 'ORF1ab', and 'ORF8' in twenty SARS-CoV-2 isolates, and evaluated their significance in protein stability and functionality in relation to virus transmission [40]. Further sequence analysis of SARS-CoV-2 isolates, of Russia and other countries has revealed a set of seven common mutations in ' $\mathrm{S}$ ' and ' $\mathrm{N}$ ' proteins, suggesting their role in varying patterns of spread [42]. Analysis of SARS-CoV-2 isolates within the United States has identified 921 mutations that included 487 missense, 348 synonymous, 66 intergenic, 4 in-frame deletions, and 5 nonsense insertions/deletions in at least three samples [48].

Recently, few genetic variants of SARS-CoV-2 with substitution mutations in 'RBD' have emerged in different geographical regions, and have subsequently spread across the borders. The UK or B.1.1.7 (501Tyr monosubstitution) and the South African or B.1.351 (20His/501Tyr dual-substitutions) strains have been suggested with much faster transmission and increased risk of death. Most recently, the Brazil or P.1 variant with three substitutions in 'RBD' and 17 other unique mutations has emerged in Brazil [49].

\section{Conclusion}

Humans lack natural immunity against zoonotic SL-CoV, notably the novel SARS-CoV-2 which has faster 'human-to-human' transmission rates and higher pathogenicity than SARS-CoV-1 and MERS-CoV Nonetheless, previous experiences with SARS-CoV-1 and MERS-CoV outbreaks have helped understanding the SARS-CoV-2 pathobiology to some extent. Over the course of cross-species infection and adaptation, some acquired mutations may lead to evolution of even more aggressive vial strains. Although the clinical manifestations of COVID-19 are well understood now, the mechanism(s) underlying its high infection rate and pathogenicity is hitherto not clearly established. In view of this, comparative genome and protein sequence analysis of SARS-CoV-2 has revealed several novel mutations, notably in the spike RBD, including generation of a furin-like site. This strongly suggests SARSCoV-2 enhanced cell attachment and facilitated entry, its high infectivity and severe pathogenesis in humans. The observed mutations within the non-structural proteins may be crucial for the enhanced replication of the viral genome. In addition, mutations within the accessory proteins could have significant roles in evading or modulating host innate immune system and sustaining virus replication. Therefore, novel mutations acquired by SARS-CoV-2 during 'human-adaptation' and 'human-to-human' spread provide insights into its transmission dynamics which together with clinical and epidemiological data can predict disease prognosis. Moreover, the consequence of these mutations on virus infectivity and tissue-tropism remain to be studied in animal models. Since SARS-CoV-2 is transmitted even during the asymptomatic phase of COVID-19, it would be interesting to study its replication in early phase, and how the innate- and adaptive-immune systems respond to its life cycle. Nonetheless, further rigorous genetic and molecular studies would enhance our knowledge on the subject towards updating treatment and preventive strategies, especially for the recently emerged variants.

\section{Declarations}

\subsection{Author Contributions}

Conceptualization, writing - original draft preparation, review and editing, M.K.P.; writing-original draft preparation, S.N. All authors have read and agreed to the published version of the manuscript.

\subsection{Funding}

The author(s) received no financial support for the research, authorship, and/or publication of this article.

\subsection{Ethical Approval}

Not applicable.

\subsection{Conflict of Interest}

The authors declare that they have no known competing financial interests or personal relationships that could have appeared to influence the work reported in this paper.

\section{References}

[1] Parvez, M. K., \& Parveen, S. (2017). Evolution and Emergence of Pathogenic Viruses: Past, Present, and Future. Intervirology, 60(1-2), 1-7. doi:10.1159/000478729.

[2] Ren, L.-L., Wang, Y.-M., Wu, Z.-Q., Xiang, Z.-C., Guo, L., Xu, T., ... Wang, J.-W. (2020). Identification of a novel coronavirus causing severe pneumonia in human: a descriptive study. Chinese Medical Journal, 133(9), 1015-1024. doi: $10.1097 / \mathrm{cm} 9.0000000000000722$. 
[3] WHO Coronavirus Disease (COVID-19) Dashboard. Available online: https://covid19.who.int/. (assessed on January 2021).

[4] Parvez, M., Jagirdar, R., Purty, R., Venkata, S., Agrawal, V., Kumar, J., \& Tiwari, N. (2020). COVID-19 pandemic: Understanding the emergence, pathogenesis and containment (Review). World Academy of Sciences Journal. 2(5). doi:10.3892/wasj.2020.59.

[5] WHO Director-General's opening remarks at the media briefing on COVID-19. Available online: https://www.who.int/dg/speeches/detail/who-director-general-s-opening-remarks-at-the-media-briefing-on-covid-19---18-march-2020. (assessed on November 2020).

[6] Seto, W., Tsang, D., Yung, R., Ching, T., Ng, T., Ho, M., ... Peiris, J. (2003). Effectiveness of precautions against droplets and contact in prevention of nosocomial transmission of severe acute respiratory syndrome (SARS). The Lancet, 361(9368), 15191520. doi:10.1016/s0140-6736(03)13168-6

[7] Van Doremalen, N., Bushmaker, T., Morris, D. H., Holbrook, M. G., Gamble, A., Williamson, B. N., ... Munster, V. J. (2020). Aerosol and Surface Stability of SARS-CoV-2 as Compared with SARS-CoV-1. New England Journal of Medicine, 382(16), 1564-1567. doi:10.1056/nejmc2004973

[8] Huang, C., Wang, Y., Li, X., Ren, L., Zhao, J., Hu, Y., ... Cao, B. (2020). Clinical features of patients infected with 2019 novel coronavirus in Wuhan, China. The Lancet, 395(10223), 497-506. doi:10.1016/s0140-6736(20)30183-5.

[9] Wong, H. Y. F., Lam, H. Y. S., Fong, A. H.-T., Leung, S. T., Chin, T. W.-Y., Lo, C. S. Y., ... Ng, M.-Y. (2020). Frequency and Distribution of Chest Radiographic Findings in Patients Positive for COVID-19. Radiology, 296(2), E72-E78. doi:10.1148/radiol.2020201160.

[10] Gu, J., Han, B., \& Wang, J. (2020). COVID-19: Gastrointestinal Manifestations and Potential Fecal-Oral Transmission. Gastroenterology, 158(6), 1518-1519. doi:10.1053/j.gastro.2020.02.054.

[11] Parvez, M. K. (2020). COVID-19 and coronaviral hepatitis: evidence of collateral damage. Future Virology, 15(6), 325-329. doi:10.2217/fvl-2020-0065.

[12] Parvez, M.K. 2020. Gastrointestinal and hepatobiliary manifestations of COVID-19: potential implications for healthcare resource-deficient countries.. Gastroenterol Hepatol Lett, 2, 7-11.

[13] Parvez, M.K. 2020. Gut feeling: The plausible faecal-oral transmission route of Covid-19. J Infect Dis Epidemiol, 6,141-143.

[14] Li, W., Shi, Z., Yu, M., Ren, W., Smith, C., Epstein, J.H., Wang, H., Crameri, G., Hu, Z., Zhang, H., Zhang, J., McEachern, J., Field, H., Daszak, P., Eaton, B.T., Zhang, S., Wang, L.F. (2005). Bats Are Natural Reservoirs of SARS-Like Coronaviruses. Science, 310(5748), 676-679. doi:10.1126/science.1118391.

[15] Schuchat, A. (2020). Public Health Response to the Initiation and Spread of Pandemic COVID-19 in the United States, February 24-April 21, 2020. MMWR. Morbidity and Mortality Weekly Report, 69(18), 551-556. doi:10.15585/mmwr.mm6918e2.

[16] Parashar, U. D. (2004). Severe acute respiratory syndrome: review and lessons of the 2003 outbreak. International Journal of Epidemiology, 33(4), 628-634. doi:10.1093/ije/dyh198.

[17] Gandhi, M., Yokoe, D. S., \& Havlir, D. V. (2020). Asymptomatic Transmission, the Achilles' Heel of Current Strategies to Control Covid-19. New England Journal of Medicine, 382(22), 2158-2160. doi:10.1056/nejme2009758.

[18] Kakodkar, P., Kaka, N., \& Baig, M. (2020). A Comprehensive Literature Review on the Clinical Presentation, and Management of the Pandemic Coronavirus Disease 2019 (COVID-19). Cureus. doi:10.7759/cureus.7560.

[19] ClinicalTrials. Available online: https://clinicaltrials.gov/ct2/ (accessed on March 2020).

[20] Parvez, M. K., \& Padhan, K. (2020). Current advances in novel SARS-CoV-2 disease (COVID-19) treatment and intervention strategies. Coronaviruses, 01. doi:10.2174/2666796701999201116125249.

[21] Chen, Y., Liu, Q., \& Guo, D. (2020). Emerging coronaviruses: Genome structure, replication, and pathogenesis. Journal of Medical Virology, 92(4), 418-423. doi:10.1002/jmv.25681.

[22] Wu, F., Zhao, S., Yu, B., Chen, Y.-M., Wang, W., Song, Z.-G., ... Zhang, Y.-Z. (2020). A new coronavirus associated with human respiratory disease in China. Nature, 579(7798), 265-269. doi:10.1038/s41586-020-2008-3.

[23] Gorbalenya, A. E., Baker, S. C., Baric, R. S., de Groot, R. J., Drosten, C., Gulyaeva, A. A., ... \& Ziebuhr, J. (2020). Coronaviridae Study Group of the International Committee on Taxonomy of Viruses. The species severe acute respiratory syndrome-related coronavirus: classifying 2019-nCoV and naming it SARS-CoV-2. Nature Microbiol, 5(4), 536-544.

[24] Ziebuhr, J. (2004). Molecular biology of severe acute respiratory syndrome coronavirus. Current Opinion in Microbiology, 7(4), 412-419. doi:10.1016/j.mib.2004.06.007. 
[25] Kumar, S., Maurya, V. K., Prasad, A. K., Bhatt, M. L. B., \& Saxena, S. K. (2020). Structural, glycosylation and antigenic variation between 2019 novel coronavirus (2019-nCoV) and SARS coronavirus (SARS-CoV). VirusDisease, 31(1), $13-21$. doi:10.1007/s13337-020-00571-5.

[26] Chan, J. F.-W., Kok, K.-H., Zhu, Z., Chu, H., To, K. K.-W., Yuan, S., \& Yuen, K.-Y. (2020). Genomic characterization of the 2019 novel human-pathogenic coronavirus isolated from a patient with atypical pneumonia after visiting Wuhan. Emerging Microbes \& Infections, 9(1), 221-236. doi:10.1080/22221751.2020.1719902.

[27] Walls, A. C., Park, Y.-J., Tortorici, M. A., Wall, A., McGuire, A. T., \& Veesler, D. (2020). Structure, Function, and Antigenicity of the SARS-CoV-2 Spike Glycoprotein. Cell, 181(2), 281-292.e6. doi:10.1016/j.cell.2020.02.058.

[28] Padhan, K., Parvez, M. K., \& Al-Dosari, M. S. (2021). Comparative sequence analysis of SARS-CoV-2 suggests its high transmissibility and pathogenicity. Future Virology. doi:10.2217/fvl-2020-0204.

[29] Wan, Y., Shang, J., Graham, R., Baric, R. S., \& Li, F. (2020). Receptor Recognition by the Novel Coronavirus from Wuhan: an Analysis Based on Decade-Long Structural Studies of SARS Coronavirus. Journal of Virology, 94(7). doi:10.1128/jvi.00127-20.

[30] Coutard, B., Valle, C., de Lamballerie, X., Canard, B., Seidah, N. G., \& Decroly, E. (2020). The spike glycoprotein of the new coronavirus 2019-nCoV contains a furin-like cleavage site absent in $\mathrm{CoV}$ of the same clade. Antiviral Research, 176, 104742. doi:10.1016/j.antiviral.2020.104742.

[31] Schoeman, D., Gordon, B., Fielding, B. C. (2021). Pathogenic Human Coronaviruses. Ref Mod Biomed Sci, B978-0-12818731-9.00052-5.

[32] Neuman, B. W., Kiss, G., Kunding, A. H., Bhella, D., Baksh, M. F., Connelly, S., ... Buchmeier, M. J. (2011). A structural analysis of $\mathrm{M}$ protein in coronavirus assembly and morphology. Journal of Structural Biology, 174(1), 11-22. doi:10.1016/j.jsb.2010.11.021.

[33] Ye, Q., West, A. M. V., Silletti, S., \& Corbett, K. D. (2020). Architecture and self-assembly of the SARS-CoV-2 nucleocapsid protein. BioRxiv. doi:10.1101/2020.05.17.100685

[34] Leung, D. T. M., Tam, F. C. H., Ma, C. H., Chan, P. K. S., Cheung, J. L. K., Niu, H., ... Lim, P. L. (2004). Antibody Response of Patients with Severe Acute Respiratory Syndrome (SARS) Targets the Viral Nucleocapsid. The Journal of Infectious Diseases, 190(2), 379-386. doi:10.1086/422040.

[35] Cárdenas - Conejo, Y., Liñan - Rico, A., García - Rodríguez, D. A., Centeno - Leija, S., \& Serrano - Posada, H. (2020). An exclusive 42 amino acid signature in pplab protein provides insights into the evolutive history of the 2019 novel human pathogenic coronavirus (SARS - CoV - 2). Journal of Medical Virology, 92(6), 688 - 692. doi:10.1002/jmv.25758

[36] Fehr, A. R., \& Perlman, S. (2015). Coronaviruses: An Overview of Their Replication and Pathogenesis. Methods in Molecular Biology, 1-23. doi:10.1007/978-1-4939-2438-7_1.

[37] Chan, J. F.-W., Kok, K.-H., Zhu, Z., Chu, H., To, K. K.-W., Yuan, S., \& Yuen, K.-Y. (2020). Genomic characterization of the 2019 novel human-pathogenic coronavirus isolated from a patient with atypical pneumonia after visiting Wuhan. Emerging Microbes \& Infections, 9(1), 221-236. doi:10.1080/22221751.2020.1719902.

[38] Ceraolo, C., \& Giorgi, F. M. (2020). Genomic variance of the 2019 - nCoV coronavirus. Journal of Medical Virology, 92(5), 522 - 528. doi:10.1002/jmv.25700.

[39] Laha, S., Chakraborty, J., Das, S., Manna, S. K., Biswas, S., \& Chatterjee, R. (2020). Characterizations of SARS-CoV-2 mutational profile, spike protein stability and viral transmission. Infection, Genetics and Evolution, 85, 104445. doi:10.1016/j.meegid.2020.104445.

[40] Benvenuto, D., Demir, A. B., Giovanetti, M., Bianchi, M., Angeletti, S., Pascarella, S., '. Cassone, A. (2020). Evidence for mutations in SARS - CoV - 2 Italian isolates potentially affecting virus transmission. Journal of Medical Virology, 92(10), 2232 - 2237. doi:10.1002/jmv.26104.

[41] Kozlovskaya, L., Piniaeva, A., Ignatyev, G., Selivanov, A., Shishova, A., Kovpak, A., ... Ishmukhametov, A. (2020). Isolation and phylogenetic analysis of SARS-CoV-2 variants collected in Russia during the COVID-19 outbreak. International Journal of Infectious Diseases, 99, 40-46. doi:10.1016/j.ijid.2020.07.024.

[42] Sheikh, J. A., Singh, J., Singh, H., Jamal, S., Khubaib, M., Kohli, S., ... Hasnain, S. E. (2020). Emerging genetic diversity among clinical isolates of SARS-CoV-2: Lessons for today. Infection, Genetics and Evolution, 84, 104330. doi:10.1016/j.meegid.2020.104330.

[43] Licastro, D., Rajasekharan, S., Dal Monego, S., Segat, L., D’Agaro, P., \& Marcello, A. (2020). Isolation and Full-Length Genome Characterization of SARS-CoV-2 from COVID-19 Cases in Northern Italy. Journal of Virology, 94(11). doi:10.1128/jvi.00543-20. 
[44] Wan, Y., Shang, J., Graham, R., Baric, R. S., \& Li, F. (2020). Receptor Recognition by the Novel Coronavirus from Wuhan: an Analysis Based on Decade-Long Structural Studies of SARS Coronavirus. Journal of Virology, 94(7). doi:10.1128/jvi.00127-20.

[45] Wrapp, D., Wang, N., Corbett, K. S., Goldsmith, J. A., Hsieh, C.-L., Abiona, O., .. McLellan, J. S. (2020). Cryo-EM structure of the 2019-nCoV spike in the prefusion conformation. Science, 367(6483), 1260-1263. doi:10.1126/science.abb2507.

[46] Cantuti-Castelvetri, L., Ojha, R., Pedro, L. D., Djannatian, M., Franz, J., Kuivanen, S., ... Simons, M. (2020). Neuropilin-1 facilitates SARS-CoV-2 cell entry and infectivity. Science, 370(6518), 856-860. doi:10.1126/science.abd2985.

[47] Shah, A., Rashid, F., Aziz, A., Jan, A. U., \& Suleman, M. (2020). Genetic characterization of structural and open reading Fram-8 proteins of SARS-CoV-2 isolates from different countries. Gene Reports, 21, 100886. doi:10.1016/j.genrep.2020.100886.

[48] Shen, L., Bard, J. D., Biegel, J. A., Judkins, A. R., \& Gai, X. (2020). Comprehensive genome analysis of 6,000 USA SARSCoV-2 isolates reveals haplotype signatures and localized transmission patterns by state and by country. doi:10.1101/2020.05.23.20110452.

[49] Emerging SARS-CoV-2 Variants. Available online: https://www.cdc.gov/coronavirus/2019-ncov/more/science-andresearch/scientific-brief-emerging-variants.html (accessed on November 2020). 